\title{
LA INCLUSIÓN EDUCATIVA EN EL CONTEXTO DE LA EDUCACIÓN CUBANA
}

\section{Educational Inclusion in the context of Cuban Education}

\author{
Evaristo Reinoso Porra, Dr. C. \\ Universidad José Martí Pérez, Cuba \\ https:// orcid.org/0000-0002-7993-8530 \\ ereinoso@uniss.edu.cu
}

\author{
Eddys Edel Ramírez Galí, MsC. \\ Universidad José Martí Pérez, Cuba \\ https:// orcid.org/0000-0003-3975-6297 \\ eddys@uniss.edu.cu
}

\begin{abstract}
Palabras claves: Atención a la Diversidad, Conducta Social, Educación es Inclusiva, Inclusión Educativa.

Recibido: 09 de julio de 2020
\end{abstract}

Keywords: Attention to Diversity, Social Behavior, Education is Inclusive, Educational Inclusion. Aceptado: 24 de septiembre de 2020

\section{RESUMEN}

La educación cubana, a partir de los logros alcanzados desde la década de los años 60 , del pasado siglo, hasta la actualidad tiene el sello del derecho, la igualdad y la justicia de todas y todos los ciudadanos, como dimensión del proyecto jurídico y político, establecido en el país, por lo antes expuesto se puede afirmar que, en Cuba, la educación es inclusiva. No obstante, se constatan deficiencias que tienen su origen en la preparación de los docentes y en la conducta social, aun alejada de los derechos y deberes de la contemporaneidad. Ante esta situación, se realiza un estudio de los aportes de las investigaciones relacionadas con la atención a la diversidad, necesidades educativas especiales e inclusión educativa; para ello se aplicaron métodos del nivel teórico (analítico-sintético, inductivo-deductivo, entre otros), en función de determinar acciones que favorecen la inclusión educativa, con el objetivo de orientar a los profesionales de la educación para la inclusión educativa que adquiere su máxima expresión en la calidad de la educación en y para la diversidad.

\begin{abstract}
Cuban education, based on the achievements of the decade of the 60 s, of the last century, up to the present has the stamp of law, equality and justice of all citizens, as a dimension of the legal and political project, established in the country, for the foregoing it can be affirmed that, in Cuba, education is inclusive. However, there are deficiencies that have their origin in the preparation of teachers and in social behavior, even far from the rights and duties of contemporaneity. Given this situation, a study is made of the contributions of research related to attention to diversity, special educational needs and educational inclusion; For this, methods of the theoretical level (analytical-synthetic, inductive-deductive, among others) were applied, in order to determine actions that favor educational inclusion, with the aim of guiding education professionals for educational inclusion that acquires its maximum expression in the quality of education in and for diversity.
\end{abstract}




\section{INTRODUCCIÓN}

La educación cubana, a partir de los logros alcanzados desde la década de los años 60, del pasado siglo, hasta la actualidad tiene el sello del derecho, la igualdad y la justicia de todas y todos los ciudadanos, como dimensión del proyecto jurídico y político, establecido en el país; además se hace corresponder con las tradiciones y realidades históricas.

A cada zona rural, por muy intrincada que estuviese, llegaba el maestro y con él o ella, la luz de la enseñanza; primero con una campaña llevada a cabo para la alfabetización del campesinado cubano, luego la construcción de escuelas sin reparar en el número de educandos que asistirían a ellas, lo importante estaba en facilitar la oportunidad, el derecho a aprender.

Reordenado el Sistema Nacional de Educación, hubo que pensar en la educación para adolescentes y jóvenes desfasados y para educandos de diferentes edades que no eran aceptados en los centros educacionales por presentar discapacidades intelectuales, sensoriales o físicas.

La atención a las personas con discapacidad se redimensiona y ocupa un lugar destacado entre las prioridades de la política educacional y social cubana; en función de ello, se habilita la elevación de la calidad de vida y la equiparación de oportunidades.

En Cuba existen leyes, decretos leyes, disposiciones, y regulaciones contenidas en los diferentes cuerpos legales como son el Código de la Niñez y la Juventud, Código de la familia, Código Civil, Código del Trabajo y Código Penal que regulan el ejercicio pleno de los derechos de estas personas sin hacer exclusión.

Se realizan diversas acciones, que como se implícita en este trabajo, asumen el objetivo de orientar a los profesionales de la educación para la inclusión educativa que adquiere su máxima expresión en la calidad de la educación en y para la diversidad; a partir de la atención a las necesidades educativas especiales y en particular a las diferencias individuales.

\section{DESARROLLO}

\section{Inclusión}

La terminología inclusión forma parte del vocabulario activo de los profesionales de la educación, en la actualidad; sería un fracaso si no pasara de ser un término de moda, que, de hecho, marca, estigmatiza y etiqueta a los educandos, a partir del uso dado como vocablo. A menudo se escuchan frases como, en la matrícula de la escuela hay educandos incluidos, y entonces cabe preguntar, ¿Cuáles son los incluidos? y la respuesta se conoce antes de escucharla, los incluidos son los educandos con discapacidad intelectual o física, los que presentan trastornos de la conducta y en la comunicación, del espectro autista o discapacidades múltiples, que comparten los salones de clases con sus coetáneos en los centros regulares.

Estos educandos, es cierto que presentan diferencias más evidentes que el resto del grupo, pero igual pudiera decirse, incluido es también, el educando muy grueso o muy delgado, el muy blanco o el muy negro, el que no deja hablar a los demás porque todo lo sabe, el que siempre esta callado por temor a ser objeto de burla y en lo sucesivo pudieran darse más características que distinguen, notablemente, a un educando del resto del grupo, entonces por naturaleza todos y todas en el grupo son incluidos.

No obstante, es prudente recurrir al término inclusión, definido por De Toro, M. y Gisbert. : 569, "como acción y resultado de incluir" y la búsqueda de este término conduce a "poner una cosa dentro de otra" y "hacer que una persona forme parte de un conjunto o colectivo"; de manera que esta última se corresponde con su aplicación en la sociedad, aun cuando requiere de otros argumentos, porque lograr que una persona forme parte de un colectivo es mucho más que integrarla, incorporarla, afilarla, adscribirla, agregarla, vincularla o asociarla a un grupo.

\section{Inclusión social}

En muchos países; de América Latina, África e incluso de Europa; se lucha por los derechos que tienen, como ciudadanos, a ser escuchados por los representantes del estado en sus reclamos de mejoras en cuanto a salud, educación, alimentación, protección y remuneración por lo que hacen; no así en Cuba que desde la década del 60, del pasado siglo, es privilegiada en cuanto a atención social, su población ha tenido los beneficios de múltiples programas que constituyen pruebas irrefutables del respeto a los derechos de cada uno de sus habitantes, sin que ninguno quede excluido, entre ellos: 
a) Programa materno infantil: incluye la atención a la mujer embarazada y el seguimiento al recién nacido.

b) Mortalidad infantil: disminuye cada año, mediante los programas materno infantil.

c) Programa Educa a tu Hijo: se aplica en las zonas de residencia por ejecutoras que a su vez son preparadas por promotoras del programa, estas últimas especialistas en edad temprana y preescolar. A las sesiones asisten niñas y niños no ubicados en círculos infantiles; el objetivo es preparar a los padres, madres y otros miembros de la familia para que puedan dar continuidad a las actividades en los hogares.

d) Programa de salud: está fundamentado bajo el principio de prevención y atención de las enfermedades y se cumple mediante el programa del médico de la familia. Juegan un papel importante los policlínicos de las comunidades y los hospitales. Todos estos recursos de forma gratuita.

e) Derecho de padres y abuelos a la licencia por maternidad: se fundamenta mediante la implementación del Programa "Maternidad y Paternidad Responsable", prepara a la pareja para el embarazo, parto, puerperio y atención del recién nacido, con énfasis en la incorporación de los hombres en estos procesos. Más tarde se les otorgo el derecho a abuelos y abuelas.

f) La casa de orientación a la familia: están ubicadas en todos los municipios del país, para ofrecer, a las familias, orientación jurídica, psicológica y pedagógica de manera gratuita.

g) Programa de Formación de Trabajadores Sociales: este programa tiene el objetivo de llegar a cada persona que lo necesite y atender, de manera diferenciada y preventiva, a cada familia.

h) Atención al adulto mayor: se materializa mediante la atención comunitaria, hospitalaria e institucional (Hogares de ancianos y casas de abuelos); la creación de centros especializados para la atención en salud al adulto mayor y la creación de las cátedras del adulto mayor.

i) Creación de canales educativos de la televisión: en su contenido tienen espacio, los programas de educación familiar.

j) Programa de Universidad para todos: concebido en función de la formación de una cultura integral de nivel universitario, mediante la televisión. El programa llega a todos los hogares cubanos y en horarios que permiten el acceso de todos y todas, sin límites de edad.

k) Programa audiovisual: consiste en la construcción de salas de televisión y video en las comunidades rurales, para el acceso de las familias y la ubicación de televisores y videos en las escuelas.

I) Programa de acceso a la literatura y la información: es contentivo de una biblioteca familiar, formada por una colección de 25 títulos de literatura cubana y universal.

m) Programa de superación integral para jóvenes. El programa abrió las puertas a jóvenes que por diferentes causas se encontraban fuera del sistema nacional de educación y tampoco se desempeñaban como trabajadores, en ocasiones por carencias académicas y en otras por no cumplir la edad laboral.

En resumen, la inclusión social, en definitiva, es un cambio de actitudes y un proceso donde la comunidad identifica y trabaja por la eliminación de las barreras, casi siempre mentales, que frenan la participación y conducen a la exclusión. En una sociedad inclusiva todos y todas son valorados sobre la base de la igualdad y la consideración hacia todos.

\section{Educación inclusiva}

En la edad temprana, los niños y las niñas asisten a los círculos infantiles, estos cuentan con especialistas que, mediante el juego, como actividad rectora, estimulan los procesos cognitivos, la comunicación y el lenguaje e inician la formación y desarrollo de hábitos, habilidades y capacidades. Los niños y las niñas que no asisten a los círculos infantiles, son atendidos por el programa Educa a tu Hijo, con objetivos similares; pero con la diferencia de un trabajo de orientación a la familia más intenso, por esta modalidad.

A los niveles educativos primaria y secundaria básica asiste el $100 \%$ de los educandos comprendidos en estas edades. No hay un solo educando fuera del sistema nacional de educación aun cuando presente una discapacidad compleja o múltiple. Para los educandos con discapacidad intelectual severa o sensorial se han creado centros educacionales con colectivos pedagógicos contentivos de especialistas que se encargan de la corrección o compensación de la discapacidad, a partir de las potencialidades individuales. Se trata de especialistas para la atención al educando con discapacidad auditiva (sordo), visual (ciego), intelectual (retraso mental) y otros. Estos educandos, después de una estancia en estos tipos de escuelas están en mejores condiciones de continuar sus estudios en los centros del nivel primario, secundario o preuniversitario. 
Los educandos con discapacidad auditiva logran el dominio de la Lengua de Señas y aprenden a comunicarse no solo con otros sordos, también, con los oyentes que comparten su salón de clases, de modo que estos últimos, estos aprenden el sistema Braille y la orientación y movilidad que comparten luego con sus coetáneos videntes.

Los educandos con discapacidades sensoriales (sordo y ciego), dificultades en el aprendizaje y trastornos de la conducta y en la comunicación continúan estudios en el nivel educativo de secundaria básica. En estos centros reciben la atención de los profesores.

En los niveles educativos de la primaria y secundaria básica se concentra el grueso de educandos con necesidades educativas especiales; en lo adelante se dispersan por los preuniversitarios, politécnicos y escuelas de oficios, generalmente, los educandos con discapacidades sensoriales.

\section{Inclusión educativa}

Los centros educacionales, también, pasan por el prisma de lo diverso, sus diferencias se evidencian en el nivel educativo; organización, planificación y dirección del trabajo educativo; preparación del colectivo pedagógico y en el tipo y estado constructivo, entre otras. De estos indicadores, es preciso detenerse en el colectivo pedagógico, pues de este depende, en gran medida, la calidad de los demás. También, por la diversidad en los docentes, aun cuando se trate de la misma institución.

Las estrategias deben responder a los preceptos inclusión educativa, a partir de considerar a esta una filosofía construida, sobre la creencia de que todas las personas son iguales y deben ser respetadas y valoradas acorde a sus derechos humanos, la inclusión es calidad educativa con equidad, es escuela, familia y sociedad donde las personas puedan encontrar en ellas lo que necesitan para desarrollarse.

Los educandos deben recibir lo que cada cual pueden asimilar, a partir de sus características individuales, requieren de una atención basada en su zona de desarrollo actual y las potencialidades de la zona de desarrollo próximo. Requieren de comprensión y una atención permeada de ternura; solo así se estaría hablando de inclusión educativa. Existen casos contrarios que recrudecen la exclusión y se manifiestan con: La etiqueta: bruto, este no aprende, nunca sabes nada, torpe; la ignorancia: está en el aula; pero como si no estuviera; la clase frontal: igual para todos, no se tiene en cuenta el diagnóstico (necesidades y potencialidades); las exigencias por encima de las potencialidades, la comunicación extraverbal: gestos de desagrado, de repulsión de impaciencia (en el caso del tartamudo), de intolerancia y el burla entre coetáneos.

Estas manifestaciones de exclusión dañan a educandos y familiares, tan es así que se registran como consecuencias casos de: Baja autoestima en el educando; rechazo al medio escolar; fugas; negativismo ante las tareas estudiantiles y agresividad o timidez.

Los directivos de la educación, maestros del nivel educativo primaria, profesores de los niveles secundaria y preuniversitario, docentes en general deben proyectarse hacia la ejecución de acciones que conduzcan a la inclusión, a partir de : Calidad en la educación; organización, planificación e impartición de clases en correspondencia con el diagnóstico individual y grupal; cumplimiento de los principios pedagógicos: unidad de lo afectivo y cognitivo; de lo instructivo y educativo, de la actividad, la comunicación y la personalidad; creatividad ante y para la solución de los problemas pedagógicos; atención a la diversidad; atención a las necesidades educativas especiales y atención a las diferencias individuales.

La inclusión educativa es calidad en la educación, entonces el modelo educativo y la escuela, tienen que proyectarse hacia el establecimiento de vínculos entre los educandos y el currículo, para que todos y todas triunfen, alcancen los objetivos de la propuesta educativa, adquieran y desarrollen estrategias de pensamientos, normas, actitudes y valores que les permitan resolver problemas de la vida cotidiana y que les preparen para aprovechar las oportunidades que la sociedad, en igualdad de acceso, posibilidades y condiciones para todos, les ofrece.

\section{Diversidad}

La diversidad humana, no escapa a las grandes, medianas y pequeñas diferencias, de ahí que se afirme, en la literatura especializada, que es un hecho real, objetivo, innegable e ineludible. Entonces, hay razones que conducen a defender las tesis que refieren que ser diferente es algo común, la diversidad es la norma y que nadie es anormal por ser diferente. 
En investigaciones recientes, López Machín, R. expresa que: "Las diferencias se evidencian, en un aprendizaje de manera diferente, con diferente ritmo y calidad, en las propias limitaciones o desventajas, que, además, son diferentes por múltiples causas (orgánico-constitucionales, fisiológicas, socio-culturales). El hecho de aprender más lentamente, con dificultades y necesitar más ayudas, no disminuye en nada la condición de seres humanos, tampoco niega la posibilidad de aprender y alcanzar el desarrollo". (López Machín, R.2014:35)

Las ideas antes expuestas, constituyen propuestas, intencionalmente, orientadas a la reflexión profunda de los profesores. Se requieren de cambios significativos en la organización, planificación y dirección del proceso educativo en las secundarias básicas.

El término de diversidad y de igualdad son principios y valores básicos, reconocidos explícitamente desde la modernidad como presupuestos para alcanzar una convivencia ordenada y articulada que gire en torno al principio de justicia. Se entiende por justicia la equidad y esta, como tal, demanda la coexistencia y el respeto a ambos principios: el de la igualdad y el de la diferencia.

El reto de la atención a la diversidad en la escuela, es un tema que ha estado presente desde los inicios mismos del desarrollo de la ciencia pedagógica, formulada en el clásico principio pedagógico de la atención a las diferencias, que encuentra su marco histórico en la Grecia antigua y que, en la actualidad, a nivel mundial, el planteamiento resulta mucho más complejo por las políticas unilaterales y las competencias por el poder.

El Sistema Nacional de Educación, en plena etapa de perfeccionamiento, se encarga del estudio de las leyes y resoluciones vigentes, que conducen a las transformaciones, de la reconceptualización de la Educación Especial, mediante su redimensionamiento y de la preparación del personal docente para asumir el reto.

\section{Necesidades educativas especiales}

El concepto de necesidades educativas está en relación con las ayudas pedagógicas y servicios educativos que determinados educandos puedan precisar para el logro del máximo crecimiento personal y social. Los educandos presentan Necesidades Educativas Especiales (NEE), cuando como consecuencia de una discapacidad o trastorno requieren ayudas o apoyos extraordinarios, para participar y avanzar en sus aprendizajes. Estos apoyos y ayudas extraordinarias pueden ser requeridos durante todas las etapas de su vida estudiantil, mientras que otros, necesitan de estas medidas por períodos específicos.

La labor de atención a las nee no resulta fácil, los resultados actuales de la práctica pedagógica demuestran avances; a partir del recorrido por un largo camino lleno de experiencias que sitúan a la educación en mejores condiciones para lograr la calidad, más justicia y que incluya a todos y a todas, el énfasis debe centrarse en la respuesta educativa que requiere el educando, y no en sus limitaciones personales.

Ajustar la respuesta educativa significa desde el punto de vista de los autores: Verdugo Alonso, M. (2003), Fernández, A. (2003), Sol Ángel Galdós Sotolongo, S. A. Y Caridad Hernández, C. (2010), Borges Rodríguez, S. A. y Orosco Delgado, M. (2013), Chkout, T. y coautores (2013), Comin, D. (2014), Guerra Iglesias, S. (2014), Terré Camacho, O. (2014) y López Machín, R. (2014) el conjunto de procesos orientados a mejorar las condiciones de aprendizaje de los educandos, el trabajo metodológico y las condiciones organizativas de los centros adicionales.

La propuesta curricular, tiene su origen y fundamentos teóricos en el currículo establecido por el ministerio de educación, y se enriquece y contextualiza, a partir de las particularidades y necesidades de los educandos, en correspondencia con la etapa educativa y a las características y demandas del contexto socio- cultural del centro. Las decisiones tomadas son relativas a: ¿Qué enseñar? Objetivos generales; ¿Cuándo? Objetivos contenidos por grado y ciclo ¿Cómo? Trabajo Metodológico y organización escolar, ¿Qué, ¿cómo y cuándo evaluar? Sistema de Evaluación (Evaluaciones sistemáticas, parciales, finales).

Las NEE se clasifican a partir de las características cognitivas psicológicas sensoriales o físicas que presentan los educandos. Por lo antes expuesto, la literatura especializada revela tipos de nee: por dificultades en el aprendizaje, discapacidades intelectuales, sensoriales o físicas, trastorno de la conducta y en la comunicación. Entre las dificultades en el aprendizaje, se reconocen por su común aparición: dislexia (dificultades en la lectura); digrafía (dificultades en la escritura) y discalculia (dificultades en el cálculo.

En la planificación del ajuste de la respuesta educativa se requiere considerar los factores de vital importancia que aseguran la unidad y coherencia de las acciones a desarrollar, entre ellos: factores curriculares, factores directivos y factores organizativos. Entre los factores curriculares se destacan por su importancia en la atención a las nee el enriquecimiento curricular, la corrección y compensación desde el currículo y las adaptaciones curriculares. 
El enriquecimiento curricular debe contribuir a acercar el proceso a una enseñanza y un aprendizaje desarrollador, coadyuvar a la superación del colectivo pedagógico, a perfeccionar su trabajo y lograr una mayor vinculación con la familia y la comunidad. Además, debe contribuir a elevar la calidad del proceso de enseñanza-aprendizaje, evitar el fracaso, propiciar el éxito de los educandos, mediante la atención a las diferencias individuales.

\section{Atención a las diferencias individuales}

Anteriormente se comentó la diversidad, su naturaleza y normalidad, además, se hizo alusión a las NEE que pueden o no estar asociadas a una discapacidad. Las nee pueden ser generalizadas o localizadas en una de las áreas del saber, o en particular en un aspecto muy puntual de una de las áreas. También, se denominan educandos con NEE, a aquellos que presentan elevadas potencialidades, denominados por algunos autores como talento, superdotado, prodigio, etc. Lo cierto es que, por una razón u otra, la mayoría de los educandos presentan nee y en consecuencia requieren de la atención a sus diferencias individuales.

Está demostrado, científicamente, que las diferencias individuales tienen una base biológica que es matizada por los componentes psicológicos y sociales; de modo que la atención a esta varía de uno a otro educando en consecuencia con las causas y características que los distingue.

La atención a las diferencias individuales, sin lugar a dudas, es la máxima expresión de la inclusión educativa, entendida esta como calidad en la educación. A partir de este fundamento se le orienta con frecuencia a los profesores la individualización de la enseñanza; pero resulta muy difícil demostrar cómo lograrlo y mucho más complejo convertirlo en práctica educativa sistemática.

Los profesores se empeñan en sistematizar la práctica educativa potencializada con la atención individualizada; pero no siempre lo logran a plenitud y se conforman con una clase frontal, algunas preguntas orales o niveles de ayudas en el puesto de trabajo. Realmente no están, suficientemente, preparados para enfrentar el desafío que le impone la enseñanza individualizada, en función de la inclusión educativa.

Se brindan algunas recomendaciones que pueden extrapolarse a las diferentes asignaturas del currículo:

Adaptar el currículo en correspondencia con el diagnostico individual y grupal. Ello exige del estudio de los componentes didácticos: objetivos, contenido, métodos y medios de enseñanza. Pensar en el triunfo del educado y evitar el fracaso.

De la misma manera que se varía el currículo, también, se organiza el tiempo o se temporaliza el contenido. Ante una nee se impone la búsqueda de tiempo, recursos y ayudas.

Se recomienda la orientación de ejercicios en sistema, tanto para la vía oral como escrita.

a) Los ejercicios en sistemas exigen de un ordenamiento por niveles de complejidad.

b) Los educandos se enfrentan a la solución del mismo sistema de ejercicios, con la diferencia que cada uno llegara hasta donde sus potencialidades se lo permitan. El profesor se sentirá satisfecho, y así se lo expresara, con los educandos que enfrentaron el reto de la zona de desarrollo próximo (ZDP), no obstante, debe sentir insatisfacción cuando un educando, por muy aventajado que sea, se queda al nivel de su zona de desarrollo actual (ZDA).

c) Se evaluará por el tránsito de la ZDA a la ZDP y en consecuencia con los resultados se premiarán a los educandos.

d) En las actividades prácticas el profesor observara, el desempeño de cada uno de los educandos en la solución del sistema de ejercicios y brindara los niveles de ayudas pertinentes.

e) Durante la aplicación de los niveles de ayudas se les respetará, a los educandos, sus individualidades y se les dará la oportunidad de pensar, reconocer el error o pedir otros niveles de ayuda.

Se recomienda la orientación de sistemas de preguntas.

a) Las preguntas aisladas o una a una, atenta contra la participación de un considerable grupo de educandos y se potencia el protagonismo de la minoría.

b) El educando debe saber, desde la base orientadora, las preguntas que puede responder, para preparase en aquellas que les resulten más cómodas o interesantes. Es importante evitar el fracaso.

c) Una vez leído el sistema de preguntas, se repite la primera, que es la de menor complejidad, para escuchar las respuestas. Se procede de igual forma en lo sucesivo. 
d) El profesor deberá tener un pronóstico de los educandos aptos para responder cada una de las preguntas, previo análisis a partir de las potencialidades.

e) El sistema está elaborado pensándose en las diferencias individuales, en las respuestas que podrá formular cada educando.

f) Una pregunta podrá tener tantas respuestas como educandos integre el grupo.

g) No se recomienda que el profesor evalúe al educando en el momento de la respuesta, para mantener la motivación por la actividad. También deberá tener en cuenta que una respuesta por más absurda que parezca tiene elementos positivos para reconocer. Es de muy mal agrado decir que una respuesta no tiene ningún valor.

h) La evaluación se recomienda para el final, no de la clase, propiamente dicha, pero si del sistema de preguntas. Tiene alto valor psicológico y pedagógico la participación de los educandos mediante la autoevaluación, coevaluación y heteroevaluacion.

i) En la evaluación, también, se aprecia la individualización de la enseñanza y no porque el más aventajado obtenga la máxima calificación y el educando con más dificultades se le otorguen calificaciones más bajas, sino porque se avalúa el tránsito de la ZDA a la ZDP.

j) La evaluación es mediadora, en la formación de la conducta; ante el hecho de saber que siempre se obtiene la más alta calificación se adoptan comportamientos que pueden ser muy nocivos por rasgos de autosuficiencia y estado de autocomplacencia y en los casos contrarios se genera la timidez, baja autoestima, el desinterés por la actividad de estudio, rechazo a la escuela y hasta comportamientos de agresividad ante la injusticia.

Las recomendaciones metodológicas, antes expuestas, requieren del acompañamiento de la paciencia, bondad, afecto y ternura, así como de las palabras de elogio y confianza.

\section{CONCLUSIÓN}

La inclusión educativa implica plantearse metas reales, aunar esfuerzos, coordinar acciones, integrar los contextos escuela-familia-comunidad, elevar la calidad de la educación. Las escuelas cubanas, en función de la inclusión educativa, asumen el reto de elevar la calidad de la educación, proyectarse hacia la atención a la diversidad y penetrar en las individualidades de los educandos, mediante la enseñanza individualizada, para alcanzar la plena atención a las necesidades educativas especiales.

\section{BIBLIOGRAFÍA}

1. Borges Rodríguez, S. A. y Orosco Delgado, M. (2013) La atención educativa a los niños, adolescentes y jóvenes con necesidades educativas especiales desde nuevos conceptos, contextos y prácticas enriquecedoras. En Chkout, T. y coautores. Sobre el perfeccionamiento de la Educación Especial. La Habana: Editorial Pueblo y Educación.

2. Borges Rodríguez, S. A. y Orosco Delgado, M. (2014) Inclusión educativa y Educación Especial: un horizonte singular y diverso para igualar las oportunidades de desarrollo. La Habana: Sello editor: Educación cubana.

3. Chkout, T. y coautores (2013) Sobre el perfeccionamiento de la Educación Especial. La Habana: Editorial Pueblo y Educación.

4. Colectivo de autores. (s/a) Enfoque y prácticas. Plataforma inclusiva para atender la diversidad en la escuela.

5. Comin, D. (2014) Transformando la educación especial en educación inclusiva. Educación. https://autismodiario.org/.../transformando-la-educacion-especial-en-edu.

6. Del Toro M. y Gisber (1968). Pequeño Larousse ilustrado. La Habana: Instituto del libro: Edición Revolucionaria.

7. Fernández, A. (2003) Educación inclusiva: enseñar y aprender entre la diversidad. Revista Digital UMBRAL www.reduc.cl.

8. Gamboa Robles, M. A., Medina Figueroa, F. L. Etelbina Mendoza Medina, E.

9. Ramírez Zaragoza, M. y Orlando Terré Camacho, O. (s/a) Docentes y prácticas inclusivas. IFODES Instituto de Formación del estado de Sonora.

10. Guerra Iglesias, S. (2014) Experiencia cubana. Atención a las necesidades educativas especiales: una aspiración de la escuela inclusiva. Copyring: Cátedra Internacional de Investigación CELAEE/AMEE.

11. López Machín, R. (2014) Educación y diversidad. Reflexiones e implicaciones metodológicas. En Guerra Iglesias. Enfoques y prácticas. Plataforma inclusiva para atender la diversidad en la escuela. Copyring: Cátedra Internacional de Investigación CELAEE/AMEE.

12. López Machín, R. (2014) Escolares con necesidades educativas especiales. La Habana: Editorial Pueblo y Educación. 
13. Martínez Nóbregas, L., Díaz Masip, M. L., Galdós Sotolongo, S. A. Y López Machín, R. (s/a) La educación inclusiva y las prácticas pedagógicas en Cuba. Retos para la preparación docente. Problemas conceptuales epistémicos y prácticos relacionados con la inclusión educativa.

14. Méndez Foros, I., Álvarez Pérez, A. E. y Borges Barrios, I. (s/a) Organización Escolar e inclusión educativa. Premisas para una escuela abierta a la diversidad.

15. Reinoso Porra, E. (s/a) La inclusión educativa en el contexto de la educación infantil en Cuba. Uniss. José Martí Pérez. Conferencia Especializada.

16. Sol Ángel Galdós Sotolongo, S. A. Y Caridad Hernández, C. (2010) La inclusión de los niños con necesidades educativas especiales. Valor metodológico del enfoque histórico-cultural. La Habana: ICCP y (UCPEJV)

17. Terré Camacho, O. (2014) Enfoque y aproximación al discurso de la diversidad en la comprensión de la inclusión educativa. En Guerra Iglesias, S. (2014)

18. Enfoques y prácticas. Plataforma inclusiva para atender la diversidad en la escuela. Copyring: Cátedra Internacional de Investigación CELAEE/AMEE.

19. Toledo Martínez, L. M., Álvarez Cruz, C. y Torres González, M. (s/a) Atención a la diversidad

20. Verdugo Alonso, M. (2003) De la segregación a la inclusión escolar. Catedrático de Psicología de la Discapacidad, Facultad de Psicología, Instituto Universitario de Integración en la Comunidad (INICO), Universidad de Salamanca.

21. Ávila, Y.; Aranda, B. y Paz, I. (2018) Estudio de Caso: Una Alternativa de Formación de la Competencia para la Orientación Educativa http://ojs.formacion.edu.ec/index.php/rif/article/view/118 DOI: https://doi.org/10.34070/rif.v6i3

22. Bell, R. (2018) Diversas Perspectivas en Relación con Algunos Aspectos Conceptuales y Prácticos de la Inclusión Educativa http://ojs.formacion.edu.ec/index.php/rif/article/view/105 DOI: https://doi.org/10.34070/rif.v6i2 